\title{
Solving Some Definite Integrals by Using Maple
}

\author{
Chii-Huei Yu \\ Department of Management and Information, Nan Jeon University of Science and Technology, Tainan City, 73746, Taiwan \\ *Corresponding Author: chiihuei@mail.njtc.edu.tw
}

Copyright (C) 2014 Horizon Research Publishing All rights reserved.

\begin{abstract}
This study uses the mathematical software Maple for the auxiliary tool to evaluate two types of definite integrals. We can obtain the closed forms of these two types of definite integrals by using differentiation with respect to a parameter and Leibniz differential rule. At the same time, we provide some definite integrals to do calculation practically. The research methods adopted in this study involved finding solutions through manual calculations and verifying these solutions by using Maple.
\end{abstract}

Keywords Definite Integrals, Closed Forms, Differentiation With Respect to a Parameter, Leibniz Differential Rule, Maple

\section{Introduction}

As information technology advances, whether computers can become comparable with human brains to perform abstract tasks, such as abstract art similar to the paintings of Picasso and musical compositions similar to those of Beethoven, is a natural question. Currently, this appears unattainable. In addition, whether computers can solve abstract and difficult mathematical problems and develop abstract mathematical theories such as those of mathematicians also appears unfeasible. Nevertheless, in seeking for alternatives, we can study what assistance mathematical software can provide. This study introduces how to conduct mathematical research using the mathematical software Maple. The main reasons of using Maple in this study are its simple instructions and ease of use, which enable beginners to learn the operating techniques in a short period. By employing the powerful computing capabilities of Maple, difficult problems can be easily solved. Even when Maple cannot determine the solution, problem-solving hints can be identified and inferred from the approximate values calculated and solutions to similar problems, as determined by Maple. For this reason, Maple can provide insights into scientific research. Inquiring through an online support system provided by Maple or browsing the Maple website (www.maplesoft.com) can facilitate further understanding of Maple and might provide unexpected insights. For the instructions and operations of
Maple, [1-7] can be adopted as references.

In calculus and engineering mathematics courses, we learnt many methods to solve the integral problems, including change of variables method, integration by parts method, partial fractions method, trigonometric substitution method, and so on. In this paper, we study the evaluation of the following two types of definite integrals which are not easy to obtain their answers using the methods mentioned above.

$$
\begin{gathered}
\int_{0}^{\pi} \frac{\cos ^{m} x}{(a+b \cos x)^{n+m+1}} d x \\
\int_{0}^{\pi / 2} \frac{\cos ^{2 p} x \cdot \sin ^{2 q} x}{\left(r+\lambda \cos ^{2} x+\beta \sin ^{2} x\right)^{n+p+q+1}} d x
\end{gathered}
$$

where $m, n, p, q$ are non-negative integers, $a, b, r, \lambda, \beta \quad$ are real numbers, and $a>|b|$, $2 r+\lambda+\beta>|\lambda-\beta|$. We can obtain the closed forms of these two types of definite integrals by using differentiation with respect to a parameter and Leibniz differential rule ; these are the main results of this paper (i.e., Theorems 1,2). The study of related integral problems can refer to [8-26]. Simultaneously, we can compare the method used in this article with that in [27]. On the other hand, we provide some definite integrals to do calculation practically. The research methods adopted in this study involved finding solutions through manual calculations and verifying these solutions by using Maple. This type of research method not only allows the discovery of calculation errors, but also helps modify the original directions of thinking from manual and Maple calculations. For this reason, Maple provides insights and guidance regarding problem-solving methods.

\section{Main Results}

Firstly, we introduce some notations and a formula used in this study.

\subsection{Notations}

(i) the $p$-th order derivative of the function $u(x)$ 
is denoted by $u^{(p)}(x)$, where $p$ is a non-negative integer.

(ii) Suppose $s, t$ are real numbers, we define $(s)_{t}=s(s-1) \cdots(s-t+1)$, and $(s)_{0}=1$.

(iii) Suppose $m, n$ are positive integers, we define

$$
\left(\begin{array}{l}
m \\
n
\end{array}\right)=\frac{m !}{n !(m-n) !} \text {, and }\left(\begin{array}{c}
m \\
0
\end{array}\right)=1 \text {. }
$$

\subsection{Integral Formula ([28])}

Suppose $a, b$ are real numbers and $a>|b|$, then the definite integral $\int_{0}^{\pi} \frac{1}{a+b \cos x} d x=\frac{\pi}{\sqrt{a^{2}-b^{2}}}$.

Next, we introduce two important theorems used in this paper.

\subsection{Differentiation With Respect to a Parameter ([29])}

Suppose $c, d, \lambda, \beta$ are real numbers and the function $f(a, x)$ is defined on $[c, d] \times[\lambda, \beta]$. If $f(a, x)$ and its partial derivative $\frac{\partial f}{\partial a}(a, x)$ are continuous functions on $[c, d] \times[\lambda, \beta]$. Then $F(a)=\int_{\lambda}^{\beta} f(a, x) d x$ is differentiable on the open interval $(c, d)$, and its derivative $\frac{d}{d a} F(a)=$ $\int_{\lambda}^{\beta} \frac{\partial f}{\partial a}(a, x) d x$ for all $a \in(c, d)$.

\subsection{Leibniz Differential Rule ([30])}

Suppose $n$ is an non-negative integer, $f(x)$ and $g(x)$ are $n$-times differentiable functions. Then the $n$-th order derivative of the product function $f(x) \cdot g(x)$,

$$
(f \cdot g)^{(n)}=\sum_{k=0}^{n}\left(\begin{array}{l}
n \\
k
\end{array}\right) f^{(k)} g^{(n-k)}
$$

The following is the first result of this study, we obtain the closed form of definite integral (1).

\subsection{Theorem 1}

Assume $m, n$ are non-negative integers, $a, b$ are real numbers, and $a>|b|$. Then the definite integral

$$
\begin{gathered}
\int_{0}^{\pi} \frac{\cos ^{m} x}{(a+b \cos x)^{n+m+1}} d x \\
=\frac{(-1)^{n} m ! n ! \pi}{(n+m) !} \times \\
\sum_{k=0}^{n} \sum_{j=0}^{m} \frac{\left(-\frac{1}{2}\right)_{k}\left(-\frac{1}{2}\right)_{n-k}\left(-\frac{1}{2}-k\right)_{j}\left(-\frac{1}{2}-n+k\right)_{m-j}}{k !(n-k) ! j !(m-j) !} \times
\end{gathered}
$$

$$
(-1)^{j} \cdot(a+b)^{-1 / 2-k-j} \cdot(a-b)^{-1 / 2-n+k-m+j}
$$

\subsubsection{Proof}

By the integral formula, we obtain

$$
\begin{gathered}
\int_{0}^{\pi} \frac{1}{a+b \cos x} d x \\
=\frac{\pi}{\sqrt{a^{2}-b^{2}}} \\
=\pi \cdot(a+b)^{-1 / 2}(a-b)^{-1 / 2}
\end{gathered}
$$

Using differentiation with respect to a parameter and Leibniz differential rule, differentiating $n$-times with respect to $a$ on both sides of (4), we have

$$
\begin{gathered}
\int_{0}^{\pi} \frac{(-1)^{n} n !}{(a+b \cos x)^{n+1}} d x \\
=\pi \cdot \sum_{k=0}^{n}\left(\begin{array}{l}
n \\
k
\end{array}\right)\left[(a+b)^{-1 / 2}\right]^{(k)}\left[(a-b)^{-1 / 2}\right]^{(n-k)} \\
=\pi \cdot \sum_{k=0}^{n}\left(\begin{array}{l}
n \\
k
\end{array}\right)\left(-\frac{1}{2}\right)_{k}\left(-\frac{1}{2}\right)_{n-k}(a+b)^{-1 / 2-k}(a-b)^{-1 / 2-n+k}
\end{gathered}
$$

Therefore,

$$
\begin{gathered}
\int_{0}^{\pi} \frac{1}{(a+b \cos x)^{n+1}} d x \\
=\frac{\pi}{(-1)^{n} n !} \cdot \sum_{k=0}^{n}\left(\begin{array}{l}
n \\
k
\end{array}\right)\left(-\frac{1}{2}\right)_{k}\left(-\frac{1}{2}\right)_{n-k}(a+b)^{-1 / 2-k}(a-b)^{-1 / 2-n+k}
\end{gathered}
$$

Also, by differentiation with respect to a parameter and Leibniz differential rule, differentiating $m$-times with respect to $b$ on both sides of (6), we obtain

$$
\begin{gathered}
(-1)^{m} \frac{(n+m) !}{n !} \cdot \int_{0}^{\pi} \frac{\cos ^{m} x}{(a+b \cos x)^{n+m+1}} d x \\
=\frac{\pi}{(-1)^{n} n !} \times
\end{gathered}
$$

$$
\begin{gathered}
\sum_{k=0}^{n}\left(\begin{array}{l}
n \\
k
\end{array}\right)\left(-\frac{1}{2}\right)_{k}\left(-\frac{1}{2}\right)_{n-k} \sum_{j=0}^{m}\left(\begin{array}{c}
m \\
j
\end{array}\right)\left[(a+b)^{-1 / 2-k}\right]^{(j)} \times \\
=\frac{(-1)^{n} \pi}{n !} \times \\
\sum_{k=0}^{n} \sum_{j=0}^{m}\left(\begin{array}{l}
n \\
k
\end{array}\right)\left(\begin{array}{c}
m \\
j
\end{array}\right)\left(-\frac{1}{2}\right)_{k}\left(-\frac{1}{2}\right)_{n-k}\left(-\frac{1}{2}-k\right)_{j}\left(-\frac{1}{2}-n+k\right)_{m-j} \times \\
(-1)^{m-j} \cdot(a+b)^{-1 / 2-k-j} \cdot(a-b)^{-1 / 2-n+k-m+j(7)}
\end{gathered}
$$

Thus, 


$$
\begin{gathered}
\int_{0}^{\pi} \frac{\cos ^{m} x}{(a+b \cos x)^{n+m+1}} d x \\
=\frac{(-1)^{n} m ! n ! \pi}{(n+m) !} \times \\
\sum_{k=0}^{n} \sum_{j=0}^{m} \frac{\left(-\frac{1}{2}\right)_{k}\left(-\frac{1}{2}\right)_{n-k}\left(-\frac{1}{2}-k\right)_{j}\left(-\frac{1}{2}-n+k\right)_{m-j}}{k !(n-k) ! j !(m-j) !} \times \\
(-1)^{j} \cdot(a+b)^{-1 / 2-k-j} \cdot(a-b)^{-1 / 2-n+k-m+j}
\end{gathered}
$$

Next, we determine the closed form of definite integral (2).

\subsection{Theorem 2}

If $n, p, q$ are non-negative integers, $r, \lambda, \beta$ are real numbers, and $2 r+\lambda+\beta>|\lambda-\beta|$. Then the definite integral

$$
\begin{gathered}
\int_{0}^{\pi / 2} \frac{\cos ^{2} x \cdot \sin ^{2 q} x}{\left(r+\lambda \cos ^{2} x+\beta \sin ^{2} x\right)^{n+p+q+1}} d x \\
=\frac{(-2)^{n+p+q} \cdot n ! \pi}{(n+p+q) !} \times \\
\sum_{k=0}^{n} \frac{\left(-\frac{1}{2}\right)_{k}\left(-\frac{1}{2}\right)_{n-k}\left(-\frac{1}{2}-k\right)_{p}\left(-\frac{1}{2}-n+k\right)_{q}}{k !(n-k) !} \times \\
(2 r+2 \lambda)^{-1 / 2-k-p} \cdot(2 r+2 \beta)^{-1 / 2-n+k-q}(8)
\end{gathered}
$$

\subsubsection{Proof}

Because

$$
\begin{gathered}
\int_{0}^{\pi / 2} \frac{1}{\left(r+\lambda \cos ^{2} x+\beta \sin ^{2} x\right)^{n+1}} d x \\
=\int_{0}^{\pi / 2} \frac{1}{\left(r+\lambda \cdot \frac{1+\cos 2 x}{2}+\beta \cdot \frac{1-\cos 2 x}{2}\right)^{n+1}} d x \\
=\int_{0}^{\pi / 2} \frac{2^{n+1}}{[(2 r+\lambda+\beta)+(\lambda-\beta) \cos 2 x]^{n+1}} d x \\
=2^{n} \cdot \int_{0}^{\pi} \frac{1}{[(2 r+\lambda+\beta)+(\lambda-\beta) \cos x]^{n+1}} d x \\
=\frac{2^{n} \pi}{(-1)^{n} n !} \cdot \sum_{k=0}^{n}\left(\begin{array}{l}
n \\
k
\end{array}\right)\left(-\frac{1}{2}\right)_{k}\left(-\frac{1}{2}\right)_{n-k}(2 r+2 \lambda)^{-1 / 2-k}(2 r+2 \beta)^{-1 / 2-n+k} \\
\left.=\frac{2^{n} \pi}{(-1)^{n}} \cdot \sum_{k=0}^{n} \frac{\left(-\frac{1}{2}\right)_{k}\left(-\frac{1}{2}\right)_{n-k}(2 r+2 \lambda)^{-1 / 2-k}(2 r+2 \beta)^{-1 / 2-n+k}}{k !(n-k) !}(6)\right)
\end{gathered}
$$

Also, using differentiation with respect to a parameter and Leibniz differential rule, differentiating $p$-times with respect to $\lambda$ on both sides of (9), we obtain

$$
\begin{gathered}
\int_{0}^{\pi / 2} \frac{\cos ^{2 p} x}{\left(r+\lambda \cos ^{2} x+\beta \sin ^{2} x\right)^{n+p+1}} d x \\
=\frac{(-2)^{n+p} \cdot n ! \pi}{(n+p) !} \cdot \sum_{k=0}^{n} \frac{\left(-\frac{1}{2}\right)_{k}\left(-\frac{1}{2}\right)_{n-k}\left(-\frac{1}{2}-k\right)_{p}}{k !(n-k) !} \times \\
(2 r+2 \lambda)^{-1 / 2-k-p} \cdot(2 r+2 \beta)^{-1 / 2-n+k}(10)
\end{gathered}
$$

Again, by differentiation with respect to a parameter and Leibniz differential rule, differentiating $q$-times with respect to $\beta$ on both sides of (10), then

$$
\begin{gathered}
\int_{0}^{\pi / 2} \frac{\cos ^{2} p \cdot \sin ^{2 q} x}{\left(r+\lambda \cos ^{2} x+\beta \sin ^{2} x\right)^{n+p+q+1}} d x \\
=\frac{(-2)^{n+p+q} \cdot n ! \pi}{(n+p+q) !} \times \\
\sum_{k=0}^{n} \frac{\left(-\frac{1}{2}\right)_{k}\left(-\frac{1}{2}\right)_{n-k}\left(-\frac{1}{2}-k\right)_{p}\left(-\frac{1}{2}-n+k\right)_{q}}{k !(n-k) !} \times \\
(2 r+2 \lambda)^{-1 / 2-k-p} \cdot(2 r+2 \beta)^{-1 / 2-n+k-q}
\end{gathered}
$$

\section{Examples}

In the following, for the two types of definite integrals in this study, we propose some examples and use Theorems 1 and 2 to determine their closed forms. On the other hand, we employ Maple to calculate the approximations of these definite integrals and their closed forms for verifying our answers.

\subsection{Example 1}

In Theorem 1, taking $m=4, n=2, a=5, b=3$, we obtain the following definite integral

$$
\begin{gathered}
\int_{0}^{\pi} \frac{\cos ^{4} x}{(5+3 \cos x)^{7}} d x \\
=\frac{\pi}{15} \cdot \sum_{k=0}^{2} \sum_{j=0}^{4} \frac{\left(-\frac{1}{2}\right)_{k}\left(-\frac{1}{2}\right)_{2-k}\left(-\frac{1}{2}-k\right)_{j}\left(-\frac{5}{2}+k\right)_{4-j}}{k !(2-k) ! j !(4-j) !} \times \\
(-1)^{j} \cdot 8^{-1 / 2-k-j} \cdot 2^{-13 / 2+k+j}(11)
\end{gathered}
$$

Using Maple to verify the correctness of (11). $>$ evalf $\left(\operatorname{int}\left((\cos (x))^{\wedge} 4 /(5+3 * \cos (x))^{\wedge} 7, x=0 . . P i\right), 14\right)$;

$$
0.0026408801975462
$$

$>$ evalf(Pi/15*sum(sum(product(-1/2-r,r=0..(k-1))*product($1 / 2-\mathrm{s}, \mathrm{s}=0 . .(1-\mathrm{k}))^{*} \operatorname{product}(-1 / 2-\mathrm{k}-\mathrm{t}, \mathrm{t}=0 . .(\mathrm{j}-1)) * \operatorname{product}(-5 / 2$ $+\mathrm{k}-\mathrm{w}, \mathrm{w}=0 . .(3-\mathrm{j})) /(\mathrm{k} ! *(2-\mathrm{k}) ! * \mathrm{j} ! *(4-\mathrm{j}) !)^{*}(-1)^{\wedge} \mathrm{j}^{*} 8^{\wedge}(-1 / 2-\mathrm{k}-\mathrm{j}) * 2$ $\wedge(-13 / 2+\mathrm{k}+\mathrm{j}), \mathrm{k}=0 . .2), \mathrm{j}=0 . .4), 14)$;

$$
0.0026408801975462
$$




\subsection{Example 2}

In Theorem 1, let $m=5, n=3, a=7, b=-2$, then the definite integral

$$
\begin{gathered}
\int_{0}^{\pi} \frac{\cos ^{5} x}{(7-2 \cos x)^{9}} d x \\
=\frac{-\pi}{56} \cdot \sum_{k=0}^{3} \sum_{j=0}^{5} \frac{\left(-\frac{1}{2}\right)_{k}\left(-\frac{1}{2}\right)_{3-k}\left(-\frac{1}{2}-k\right)_{j}\left(-\frac{7}{2}+k\right)_{5-j}}{k !(3-k) ! j !(5-j) !} \times \\
(-1)^{j} \cdot 5^{-1 / 2-k-j} \cdot 9^{-17 / 2+k+j}
\end{gathered}
$$

Next, we use Maple to verify the correctness of (12).

$>$ evalf $\left(\operatorname{int}\left((\cos (x))^{\wedge} 5 /(7-2 * \cos (x))^{\wedge} 9, x=0 . . P i\right), 14\right)$;

$$
2.1552539566445 \cdot 10^{-7}
$$

$>$ evalf(-Pi/56*sum(sum(product(-1/2-r,r=0..(k-1))*product ( $-1 / 2-\mathrm{s}, \mathrm{s}=0 . .(2-\mathrm{k})) * \operatorname{product}(-1 / 2-\mathrm{k}-\mathrm{t}, \mathrm{t}=0 . .(\mathrm{j}-1)) * \operatorname{product}($ $-7 / 2+\mathrm{k}-\mathrm{w}, \mathrm{w}=0 . .(4-\mathrm{j})) /(\mathrm{k} ! *(3-\mathrm{k}) ! * \mathrm{j} ! *(5-\mathrm{j}) !) *(-1)^{\wedge} \mathrm{j}^{*} 5^{\wedge}(-1 / 2-\mathrm{k}-$ j)*9^(-17/2+k+j),k=0..3),j=0..5),14);

\section{$2.1552539566444 \cdot 10^{-7}$}

\subsection{Example 3}

In Theorem 2, let $n=4, p=2, q=1, r=2, \lambda=3, \beta=2$, we can determine the definite integral

$$
\begin{gathered}
\int_{0}^{\pi / 2} \frac{\cos ^{4} x \cdot \sin ^{2} x}{\left(2+3 \cos ^{2} x+2 \sin ^{2} x\right)^{8}} d x \\
=\frac{-64 \pi}{105} \cdot \sum_{k=0}^{4} \frac{\left(-\frac{1}{2}\right)_{k}\left(-\frac{1}{2}\right)_{4-k}\left(-\frac{1}{2}-k\right)_{2}\left(-\frac{9}{2}+k\right)_{1}}{k !(4-k) !} \cdot 10^{-5 / 2-k} \cdot 8^{-11 / 2+k}
\end{gathered}
$$

$>\operatorname{evalf}\left(\operatorname{int}\left(((\cos (\mathrm{x})))^{\wedge} 4 *(\sin (\mathrm{x}))^{\wedge} 2\right) /\left(2+3 *(\cos (\mathrm{x}))^{\wedge} 2+2 *(\sin (\right.\right.$ $\left.\left.\left.\mathrm{x}))^{\wedge} 2\right)^{\wedge} 8, \mathrm{x}=0 . . \mathrm{Pi} / 2\right), 14\right)$;

\section{$5.1015015791996 \cdot 10^{-7}$}

$>$ evalf(-64*Pi/105*sum(product(-1/2-r,r=0..(k-1))*product( $-1 / 2-\mathrm{s}, \mathrm{s}=0 . .(3-\mathrm{k})) * \operatorname{product}(-1 / 2-\mathrm{k}-\mathrm{t}, \mathrm{t}=0 . .1) *(-9 / 2+\mathrm{k}) /(\mathrm{k}$ ! $*(4-$ $\left.\left.\mathrm{k}) !)^{*} 10^{\wedge}(-5 / 2-\mathrm{k}) * 8^{\wedge}(-11 / 2+\mathrm{k}), \mathrm{k}=0 . .4\right), 14\right)$;

$$
5.1015015791995 \cdot 10^{-7}
$$

\subsection{Example 4}

In Theorem 2, if $n=3, p=2, q=4, r=5, \lambda=-2, \beta=4$, then the definite integral

$$
\begin{gathered}
\int_{0}^{\pi / 2} \frac{\cos ^{4} x \cdot \sin ^{8} x}{\left(5-2 \cos ^{2} x+4 \sin ^{2} x\right)^{10}} d x \\
=\frac{-8 \pi}{945} \cdot \sum_{k=0}^{3} \frac{\left(-\frac{1}{2}\right)_{k}\left(-\frac{1}{2}\right)_{3-k}\left(-\frac{1}{2}-k\right)_{2}\left(-\frac{7}{2}+k\right)_{4}}{k !(3-k) !} \cdot 6^{-5 / 2-k} \cdot 18^{-15 / 2+k}
\end{gathered}
$$

$>\operatorname{evalf}\left(\operatorname{int}\left(\left((\cos (\mathrm{x}))^{\wedge} 4 *(\sin (\mathrm{x}))^{\wedge} 8\right) /\left(5-2 *(\cos (\mathrm{x}))^{\wedge} 2+4 *(\sin (\mathrm{x}\right.\right.\right.$ )$\left.\left.\left.)^{\wedge} 2\right)^{\wedge} 10, \mathrm{x}=0 . . \mathrm{Pi} / 2\right), 14\right)$;

\section{$2.7089130065827 \cdot 10^{-10}$}

$>$ evalf(-8* $\mathrm{Pi} / 945 *$ sum(product(-1/2-r, $\mathrm{r}=0$...(k-1))*product($1 / 2-\mathrm{s}, \mathrm{s}=0 . .(2-\mathrm{k}))^{*} \operatorname{product}(-1 / 2-\mathrm{k}-\mathrm{t}, \mathrm{t}=0 . .1) * \operatorname{product}(-7 / 2+\mathrm{k}-\mathrm{w}$ $\left.\left., \mathrm{w}=0 . .3) /(\mathrm{k} ! *(3-\mathrm{k}) !)^{*} 6^{\wedge}(-5 / 2-\mathrm{k}) * 18^{\wedge}(-15 / 2+\mathrm{k}), \mathrm{k}=0 . .3\right), 14\right)$;

\section{$2.7089130065828 \cdot 10^{-10}$}

\section{Conclusion}

In this paper, we provide a new technique to solve two types of definite integrals, and we know that differentiation with respect to a parameter and Leibniz differential rule play significant roles in the theoretical inferences of this study. In fact, the applications of these two theorems are extensive, and can be used to easily solve many difficult problems; we endeavor to conduct further studies on related applications. On the other hand, Maple also plays a vital assistive role in problem-solving. In the future, we will extend the research topic to other calculus and engineering mathematics problems and solve these problems by using Maple. These results will be used as teaching materials for Maple on education and research to enhance the connotations of calculus and engineering mathematics.

\section{REFERENCES}

[1] F. Garvan, The Maple Book, London: Chapman \& Hall/CRC, 2001.

[2] M. L. Abell and J. P. Braselton, Maple by Example, 3rd ed., New York: Elsevier Academic Press, 2005.

[3] D. Richards, Advanced Mathematical Methods with Maple, New York: Cambridge University Press, 2002.

[4] C. Tocci and S. G. Adams, Applied Maple for Engineers and Scientists, Boston: Artech House, 1996.

[5] J. S. Robertson, Engineering Mathematics with Maple, New York: McGraw-Hill, 1996.

[6] R. J. Stroeker and J. F. Kaashoek, Discovering Mathematics with Maple : An Interactive Exploration for Mathematicians, Engineers and Econometricians, Basel: Birkhauser Verlag, 1999.

[7] C. T. J. Dodson and E. A. Gonzalez, Experiments in Mathematics Using Maple, New York: Springer-Verlag, 1995.

[8] A. A. Adams, H. Gottliebsen, S. A. Linton, and U. Martin, Automated theorem proving in support of computer algebra: symbolic definite integration as a case study, Proceedings of the 1999 International Symposium on Symbolic and Algebraic Computation, pp. 253-260, Vancouver, Canada, 1999.

[9] C. Oster, Limit of a definite integral, SIAM Review, Vol. 33, No. 1, pp. 115-116, 1991.

[10] M. A. Nyblom, On the evaluation of a definite integral 
involving nested square root functions, Rocky Mountain Journal of Mathematics, Vol. 37, No. 4, pp. 1301-1304, 2007.

[11] C. -H. Yu, Using Maple to study two types of integrals, International Journal of Research in Computer Applications and Robotics, Vol. 1, Issue. 4, pp. 14-22, 2013.

[12] C. -H. Yu, A study on integral problems by using Maple, International Journal of Advanced Research in Computer Science and Software Engineering, Vol. 3, Issue. 7, pp. 41-46, 2013.

[13] C.-H. Yu, Application of Maple on evaluation of definite integrals, Applied Mechanics and Materials, Vols. 479-480 (2014), pp. 823-827, 2013.

[14] C. -H. Yu, Solving some integrals with Maple, International Journal of Research in Aeronautical and Mechanical Engineering, Vol. 1, Issue. 3, pp. 29-35, 2013.

[15] C. -H. Yu, Evaluating some integrals with Maple, International Journal of Computer Science and Mobile Computing, Vol. 2, Issue. 7, pp. 66-71, 2013.

[16] C. -H. Yu, The application of Parseval's theorem to integral problems, Applied Mathematics and Physics, Vol. 2, No. 1, pp. 4-9, 2014.

[17] C. -H. Yu and B. -H. Chen, Solving some types of integrals using Maple, Universal Journal of Computational Mathematics, in press.

[18] C. -H. Yu, Studying three types of integrals with Maple, American Journal of Computing Research Repository, in press.

[19] C. -H. Yu, Application of Parseval's theorem on evaluating some definite integrals, Turkish Journal of Analysis and Number Theory, in press.

[20] C. -H. Yu, Using Maple to study the integrals of trigonometric functions, Proceedings of the 6th
IEEE/International Conference on Advanced Infocomm Technology, No. 00294, 2013.

[21] C.-H. Yu, Application of Maple on some type of integral problem, Proceedings of the Ubiquitous-Home Conference 2012, pp.206-210, 2012.

[22] C.-H. Yu, Application of Maple on evaluating the closed forms of two types of integrals, Proceedings of the 17th Mobile Computing Workshop, ID16, 2012.

[23] C. -H. Yu, A study of the integrals of trigonometric functions with Maple, Proceedings of the Institute of Industrial Engineers Asian Conference 2013, Springer, Vol. 1, pp. 603-610, 2013.

[24] C.-H. Yu, Application of Maple on some integral problems, Proceedings of the International Conference on Safety \& Security Management and Engineering Technology 2012, pp. 290-294, 2012.

[25] C.-H. Yu, Application of Maple: taking two special integral problems as examples, Proceedings of the 8th International Conference on Knowledge Community, pp.803-811, 2012.

[26] C.-H. Yu, Application of Maple on the integral problem of some type of rational functions, Proceedings of the Annual Meeting and Academic Conference for Association of IE, D357-D362, 2012.

[27] Differentiation under the integral sign, online available from http://en.wikipedia.org/wiki/Differentiation_under_the_integ ral_sign

[28] D. Zwillinger (Ed.), CRC Standard Mathematical Tables and Formulae, London: Chapman \& Hall/CRC, p451, 2003.

[29] L. Flatto, Advanced Calculus, Baltimore: The Williams \& Wilkins, p405, 1976.

[30] T. M. Apostol, Mathematical Analysis, 2nd ed., Boston: Addison-Wesley, p121, 1975. 